\title{
Análisis de las técnicas constructivas medievales de la Valdinievole (Toscana): desde la madera al ladrillo
}

\author{
Juan Antonio Quirós Castillo *
}

\section{INTRODUCCIÓN}

El objetivo del presente artículo es el de presentar brevemente algunas problemáticas relativas a las modificaciones de las técnicas constructivas en el período medieval, valorando su significado e incidencia en la historia de la sociedad. No se presentará una síntesis de las diversas técnicas empleadas o un catálogo de edificios, sino más bien se reflexionará sobre algunos problemas relativos a la introducción de nuevos conocimientos técnicos en la construcción de edificios; algunos de ellos se habían perdido con el final del mundo clásico, y otros aparecen en la edad media y son completamente nuevos. Son cambios radicales en la historia de la cultura material, que actúan como indicadores de procesos más amplios y de transformaciones profundas ${ }^{2}$.

En concreto, observaremos los cambios relativos al empleo de los materiales de construcción, desde la madera hasta el ladrillo, analizando los contextos históricos en los que se produce su sustitución, y las motivaciones técnicas y sociales que están en la base de las mismas.

Más que referirse a espacios muy amplios considerando datos aislados provenientes de zonas distantes, nos ha parecido más conve- niente analizar un caso específico de forma exhaustiva, pero haciendo frecuentes referencias a un contexto amplio, en este caso la Toscana medieval, con el fin de superar el mero análisis local y el particularismo del caso específico. Para ello hemos elegido un área rural, en la que es posible y necesario desplegar toda la riqueza informativa de las fuentes arqueológicas debido a la debilidad del registro documental.

El área ilustrada es la Valdinievole en la Toscana septentrional, donde desde hace cuatro años se realiza un proyecto de arqueología global (MILANESE, QUIRÓS CASTILLO 1993), entendiendo como tal el estudio sistemático del territorio con un carácter diacrónico (desde la prehistoria hasta el siglo actual), empleando metodologías de estudio muy variadas (desde la prospección a las intervenciones programadas y de urgencia, combinadas con la arqueología de la arquitectura y el estudio de las fuentes indirectas), y temáticas amplias (arqueología urbana, historia de la cultura material, arqueología de la producción, arqueología del poblamiento rural y del territorio) ${ }^{3}$. El objetivo último es elaborar una historia del territorio a partir de las fuentes materiales. En este contexto se pueden hacer las siguientes reflexiones sobre las técnicas constructivas medievales.

\footnotetext{
* Becario pre-doctoral EEHAR - Roma (C.S.I.C.)

I El presente artículo recoge el texto de un Seminario celebrado en la Escuela Española de Historia y Arqueología de Roma el 2 I de Febrero de 1996.

2 Los principales estudios sobre estos procesos BOUGARD, HUBERT, NOYÉ 1987; FRANCOVICH, CUCINI, PARENTI 1989.

3 Sobre el concepto de arqueología global MANNONI, CABONA, FERRANDO 1988.
} 
Antes de seguir, intentaremos caracterizar geográfica e históricamente el área estudiada, con el fin de ofrecer algunas claves de lectura imprescindibles para nuestro propósito. La Valdinievole (fig. I) (que podría traducirse como el valle del río Nievole, aunque en realidad recoge igualmente las cuencas de los ríos Pescia, Borra y otros de menor entidad), puede considerarse como una región histórica que ha funcionado de forma unitaria en todo el período postclásico, si bien nunca ha formado una unidad política concreta; son los límites físicos los que definen su extensión (QUIRÓs CASTILLO 1996c). Ocupa un espacio de unos $428 \mathrm{Km}$. cuadrados, y está delimitada por el río Arno al sur, la sierra de Monte Albano al este (que la separa de la llanura de Pistoia), al norte el preapenino toscoemiliano (llegando a superar los 1.100 m.), y las colinas de Cerbaia al oeste (que la separan de la llanura de Lucca) (PUCCINELLI 1970).

Morfológicamente se puede dividir en tres espacios distintos; la baja Valdinievole en el sector meridional, que se encuentra por debajo de los $100 \mathrm{~m}$. y ha estado ocupada durante toda la edad media por un pantano desecado casi totalmente en el siglo XVIII; una franja colinar (entre los 100 y $300 \mathrm{~m}$.) donde se disponen gran parte de los principales centros habitados medievales, y una zona de montaña (entre 300 y I I00 m.), ubicada en el sector septentrional.

A la presencia de este pantano, que determina geográficamente toda el área, hay que añadir otro factor de gran importancia en la organización del territorio: la ausencia de centros urbanos que hayan articulado el poblamiento. La presencia a menos de $15-18 \mathrm{Km}$. de ciudades como Lucca y Pistoia, ya de gran peso en el período romano, les ha llevado a ejercer una fuerte influencia sobre este área. Este peso de los centros urbanos perdurará más allá de la caída del imperio y las invasiones bárbaras. En este sentido, la Valdinievole se integra en el área de control de la ciudad de Lucca, que constituye la sede de un Ducado lombardo de gran importancia, fortalecido por la llegada de los carolingios en el siglo IX (MANCINI 1986).

Es en este contexto en el que el potente obispo de Lucca y la aristocracia que nacerá bajo su control, extienden sus intereses en nuestro valle durante los siglos anteriores al año 1000. En este mismo período se funda en la ciudad de Lucca el marquesado de Toscana, consolidando la posición de esta ciudad como verdadera capital de toda la región. Con el siglo XI tiene lugar la formación de señorías territoriales por parte de la aristocracia terrateniente en el campo, ya que tienden a romper sus lazos con la ciudad. En cambio, en los centros urbanos, el crecimiento de nuevos grupos comerciales y nobiliarios llevan a la formación de ciudades-estado autónomas (los comune) a finales del mismo siglo, que entrarán inmediatamente en conflicto con las señorías rurales. Así, tiene lugar en la Valdinievole durante los siglos XII y XIII la expansión progresiva del área controlada por el nuevo comune de Lucca, que consigue contrastar el peso de estas señorías hasta el año 1339, en que la República de Florencia termina por conquistar las dos terceras partes del territorio de la Valdinievole.

La última nota ambiental que conviene considerar es que la Valdinievole presenta potencialmente una gran riqueza de recursos capaces de ser empleados como materiales constructivos; en la franja septentrional aflora la formación litológica llamada falda toscana, dominio casi exclusivo de la arenisca y de otras piedras adecuadas para la construcción, combinada con calizas con las cuales se preparan las cales de las argamasas; igualmente, en los bosques montanos se encuentra abundante madera a disposición; por último en toda la llanura sur afloran limos y arcillas villafranquianas aluviales óptimas para la preparación de ladrillos.

Es, pues, en este contexto espacial en el que intentaremos encuadrar algunas problemáticas relativas a las transformaciones de las técnicas constructivas y su significado histórico.

\section{El abandono de las técnicas cons- tructivas tardoantiguas y la elaboración de un lenguaje altomedieval}

Éste es uno de los períodos peor documentados arqueológicamente en la Valdinievole. La falta de secuencias estratigráficas e inclu- 
so la ausencia de instrumentos útiles para la comprensión de este período (como son los materiales datables con claridad entre los siglos que van desde las últimas importaciones norteafricanas al X), no permiten conocer el ritmo de las modificaciones de las técnicas constructivas.

En un solo yacimiento, la villa romana de Vaiano (MILANESE et alii 1996), se documenta la continuidad de uso desde la época tardorromana hasta la baja edad media ${ }^{4}$. Objeto de excavaciones de urgencia en el año 1985, se pudo documentar como a la primera fase correspondiente a una villa altoimperial, se sucedía un oratorio en el que apareció un mosaico del siglo V. A este edificio aparecen asociadas tumbas "alla capuccina" (con tégulas a doble vertiente), presumiblemente de los siglos IV-V.

Sobre esta última fase, se han individualizado restos de un fondo de cabaña datado en forma genérica en el altomedievo (entre los siglos $\vee$ y $X$ ). La presencia de diversos agujeros de poste evidencian la existencia de, al menos, una cabaña de madera, de la que no ha sido posible reconstruir su planta o definir sus características constructivas; a la escasa consistencia del material hay que sumar la influencia de las obras realizadas durante la construcción en el siglo $X$ de una nueva iglesia dedicada a S. Lorenzo justo sobre la cabaña.

De todas formas, la tendencia dominante en el período tardorromano y altomedieval es el abandono progresivo de las zonas bajas que ocupaban los yacimientos imperiales, y la progresiva conquista de áreas colinares elevadas; yacimientos como Puntallo o Petano documentan bien este proceso. Son asentamientos muy pobres, que proporcionan una cantidad de cerámica muy pequeña, y de la que, por las prospecciones, no conocemos la entidad de sus estructuras. Otros yacimientos como Fontanaccio de Medicina se abandonan en el siglo $\mathrm{V}$ (QUIRÓS CASTILLO 1996).

Los yacimientos datados de forma genérica en los siglos de la alta edad media confir- man la morfología de las estructuras constructivas ya vistas en el caso de la fase III de Vaiano: aparecen cabañas de madera, raramente con zócalo en piedra y alzado de madera, y los restos son tan poco consistentes, que impiden una interpretación planimétrica de las estructuras. Se trata de una arquitectura rural, pobre y funcional, por lo que no se documentan las tendencias generalmente presentes en edificios de cierto prestigio (torres o iglesias), ni tan siquiera la reutilización de material romano.

Un primer ejemplo de estas cabañas en madera ha sido recuperado en 1992 en la ciudad de Pescia, en la plaza S. Romualdo, donde se ha podido identificar una primera fase de ocupación del área con la presencia de una cabaña de forma elíptica, realizada con 8 agujeros de palo, y que en parte ha sido destruida por una riada posiblemente en algún momento del siglo $X$.

Igualmente, en la primera fase de Terrazzana se puede observar una situación similar. Se trata de una pequeña aldea medieval, objeto de excavaciones sistemáticas desde el año 1992 , donde se han podido identificar fondos de cabañas anteriores a los siglos XI-XII. Ubicada en el sector septentrional de la Valdinievole, puede considerarse como un ejemplo muy significativo del modelo de poblamiento dominante en los últimos siglos altomedievales, en el período lombardo y carolingio.

Los restos más significativos pertenecientes a esta aldea se encuentran en la cima del poblado, en la cabeza de una terraza fluvial excavada por el río Pescia. En esta zona se encuentran los restos de agujeros de postes muy profundos (hasta $90 \mathrm{~cm}$.), que definen una estructura elíptica, que en parte ha sido alterada y destruida por la construcción de las fases siguientes. El aspecto quizás más interesante de las estructuras de este espacio dominante y privilegiado del poblado, es la presencia de una muralla o muro de cierre que delimitaba el espacio habitado. Esta muralla ha sido realizada con un zócalo (conservado en dos hiladas) realizado con bloques de arenisca local, ape-

4 Sobre la continuidad del poblamiento romano en Toscana, CAMBI I993; CIAMPOLTRINI 1995. 
nas regularizados, y alternados con nuevos agujeros de palos de notables dimensiones, que debían alojar las estructuras portantes del alzado de dicho muro. La dirección de tal muro es radicalmente distinta a la muralla perteneciente a la segunda fase (y de la que se hablará posteriormente), testimoniando la diacronía.

Otros fondos de cabañas se han encontrado en otra terraza inferior, que testimonian la extensión del poblado, que se reduce a pocas unidades familiares.

Otros casos similares son los del la primera fase del castillo de Montecatini, las cabañas del yacimiento de Valle Cauria (QUIRÓS CASTILLO 1996a) o el caso de Fucecchio (VANNI DESIDERI 1987).

Recapitulando, se puede observar una ruptura radical en el ámbito de las técnicas constructivas entre el período tardoantiguo y el altomedievo, con el abandono de las estructuras en piedra y el uso exclusivo de la madera. No hay que pensar que en el período romano o tardoantiguo no se construyesen también cabañas de madera, pero ahora estas construcciones son las únicas presentes, y además elaboran modelos morfológicamente autónomos que pertenecen a un lenguaje altomedieval (BROGIOLO 1994). Esto se relaciona, en la Valdinievole, con un cambio radical de los modelos de poblamiento y de estructuración y organización del territorio. Se abandonan las áreas del fondo del valle, donde se había organizado desde el período altoimperial una red de villae y vicus menores, y se reconquista la colina, antes ocupada ya por los yacimientos etruscos. El proceso parece iniciarse en época teodosiana, cuando en la zonas de montaña se comienzan a ocupar las zonas más fértiles del valle (QUIRÓs CASTILLO 1996a). Las técnicas constructivas se caracterizan por el empleo directo de los recursos que ofrece la naturaleza del valle, bosques y afloramientos de arenisca, que apenas vienen modificados después de la extracción. La antropización del paisaje de montaña, considerado antes marginal, es aún bastante limitada, y por los datos con que contamos parece que se produce una integración con los recursos naturales de forma equilibrada. Las fuentes documentales con las que contamos muestran la "compenetración existente entre el hombre y la naturaleza" (ANDREOLLI 1983, p. 13), de la que la alimentación puede ser un indicador significativo; la dieta campesina en este período es muy variada y articulada, combinando caza, recolección, cultivo, pesca, etc. de una forma tal que desaparecerá completamente en la baja edad media, cuando se imponga la especialización productiva, las limitaciones feudales y la creación de mercados regionales (MONTANARI 1979). A estas variables hay que añadir el hecho de que las casas eran realizadas por los propios campesinos, no documentándose, salvo en la ciudad o en el caso de edificios de un cierto prestigio (iglesias, castillos, etc.), artesanos especializados.

\section{El paso de las cabañas de madera a las estructuras en piedra}

Un momento transcendental en la historia de las técnicas constructivas medievales es el de la sustitución en el ámbito de la arquitectura rural, a partir del año 1000, de la madera por la piedra como material constructivo (PESEZ 1985; FRANCOVICH, CUCINI, PARENTI 1989). El uso de la piedra como material constructivo no había desaparecido, pero si había quedado relegado a la ciudad y a una serie de estructuras que podemos llamar "de prestigio". De hecho, la presencia de maestros especialistas en el trabajo de la piedra se puede documentar en la ciudad de Lucca ya en el período lombardo tardío y carolingio, y en los documentos abundan las referencias a estas construcciones a partir de los siglos IX y X (VIOLANTE 1987).

La situación en la Valdinievole es muy compleja, ya que a partir del siglo $X \mid$ se comienzan a configurar toda una serie de formas de hábitat muy articuladas y jerarquizadas, que explican la diversidad de las técnicas y los materiales constructivos empleados.

Un ejemplo muy significativo de este proceso es el castillo de Terrazzana, del que ya se ha hablado. En la segunda fase del poblado se produce la radical sustitución de las técnicas constructivas. El recinto amurallado con zócalo en piedra y alzado en madera, viene susti- 
tuido por una nueva cinta de $90 \mathrm{~cm}$. de espesor, realizada con bloques de arenisca regularizados y dispuestos en hiladas horizontales y paralelas, conservadas hasta I metro de altura (fig. 2). En cambio, las cabañas de madera son sustituidas por casas en piedra de dos pisos, construidas con mampuestos de arenisca que forman hiladas subhorizontales o irregulares, con el empleo frecuente de cuñas.

Es de vital interés para comprender el significado de tales construcciones, el análisis de ciertas características técnicas de estos paramentos. En el caso de la muralla antes descrita, vemos como se trata de un muro que presenta un núcleo poco configurado, compuesto por dos hiladas externas de bloques regulares bien trabajados y un emplecton realizado con los desechos que se forman como fruto de la elaboración del material. La argamasa no se emplea nunca. A estas particularidades hay que añadir la utilización de nuevos instrumentos para la conformación y el acabado de los sillares (polka y el scalpello), ausentes completamente no sólo en la primera fase de Terrazzana, sino también en toda la Valdinievole antes del siglo $X I^{5}$. Todos estos elementos nos hablan de la introducción de una tecnología alóctona, de la importación de mano de obra especializada con el fin de construir esta muralla ${ }^{6}$. La presencia de estas murallas construidas sin argamasa se documenta también en otras zonas de Lucca, como en el caso del castillo de poggio S. Martino, construido en el año I I07, y cerrado por un recinto amurallado de una altura de I, I 8- I,77 m según los documentos (SETTIA 1984, p.368; BERTINI I8I8, n. 96 p.|26-7).

Como confirmación de esta interpretación, hay que señalar la presencia de una marca de cantero (fig. 3), en este caso una simple cruz incisa en la misma muralla, que son extremamente raras en toda la Toscana medieval (BIANCHI 1993).
Esta técnica contrasta completamente con la que se emplea en la construcción de las casas (fig. 4). Como se decía antes, son casas con planta rectangular de $6 \times 8 \mathrm{~m}$., construidas en dos pisos que se apoyan sobre la colina, cortada y regularizada a forma de escalera sobre la que descansan las estructuras del piso inferior. Este nivel inferior ha sido interpretado como un espacio empleado como cuadras para animales de pequeña talla (cerdos, cabras, ovejas). Frecuentemente aparece dividido en dos ambientes, pudiendo funcionar uno de ellos como almacén. En el nivel superior del aterrazamiento se coloca la cocina y el espacio de residencia, probablemente construido en madera encima de la cuadra.

Esta tipología constructiva se repite de forma monótona en todo el yacimiento, y las sucesivas reconstrucciones, realizadas cada 2-4 generaciones, mantienen fundamentalmente las mismas pautas constructivas ${ }^{7}$.

La técnica constructiva de estas casas es bastante pobre, realizada con mampuestos poco elaborados, apenas colocados en el muro una vez extraídos de la cantera situada in loco, regularizados al máximo con mazas o hachas, y sin signos de acabado, tan frecuentes en la muralla coetánea. Son muros de unos $50 \mathrm{~cm}$. de espesor, con un núcleo poco configurado y sin argamasa. Presentan las mismas características de casas de otros castillos de la Valdinievole (como Lignana, QUIRÓs CASTILLO 1996 O Verruca), o Apenínicos (Monte Zignago, FERRANDO, GARDINI, MANNONI 1978), donde la argamasa no aparecerá hasta el siglo XIV, permitiendo alcanzar los 3 y 4 pisos, pero sin variar el modelo arquitectónico.

Todos estos datos pueden indicar que las casas presentes en aldea son un producto realizado por los propios habitantes de Terrazzana, personas carentes de los instrumentos

\footnotetext{
5 Sobre los instrumentos de trabajo de la piedra BESSAC 1986; para el caso específico de la Toscana en época medieval BIANCHI, PARENTI 1991.

6 Sobre la introducción de técnicas constructivas y sus pervivencias y transformaciones sucesivas, es iluminante el caso de la Roca de San Silvestro, BIANCHI 1995.

7 Se trata de un modelo de casa apoyado en la pendiente, "casa de pendio", que caracteriza la arquitectura apenínica durante toda la edad media y parte del postmedievo (QUIROS CASTILLO, ZANCHETTA' 1994).
} 
empleados en las murallas, y que perpetúan las morfologías constructivas más allá del mismo momento fundacional del castillo en el siglo $\mathrm{XI}-\mathrm{XIl}$; así, las reconstrucciones se realizan repitiendo el mismo modelo arquitectónico, indicando que se trata de una tecnología adquirida y mantenida por el grupo.

En el caso de Terrazzana, los cambios en las técnicas constructivas son un elemento indicador de gran importancia de los cambios que tienen lugar en el paisaje rural de la Valdinievole de los siglos XI y XII. Se asiste a un proceso de reorganización feudal del paisaje agrario, apenas acentuado en la zona meridional donde la presencia de curtes había ya realizado esta labor en los siglos VIII-X (WICKHAM 199I), y más marcado en la zona norte montañosa donde se ubica Terrazzana, área más abierta y menos atractiva a los señores.

Este proceso tiene como resultado más evidente la simplificación y selección del poblamiento, así como la sustitución de la madera por la piedra, incluso en centros ya fortificados como era Terrazzana I. De esta manera, la presencia de artesanos llamados desde el exterior para renovar la arquitectura urbana demuestran una intervención señorial directa en el proceso de incastellamento. Su nueva muralla es el elemento principal de organización del nuevo poblado, y es más amplia, sólida y simbólica de lo que podía ser el primer recinto realizado en madera con zócalo en piedra. Y es aquí en este aspecto en el que podemos hablar de "incastellamento" feudal y no en la mera aparición de un recinto amurallado, que ya existía anteriormente, pero que había sido construido por los propios habitantes de las cabañas del hábitat altomedieval empleando técnicas y materiales constructivos para ellos más familiares y accesibles.

La presencia de este maestro anónimo, presente a través de su signo lapidario, responde a un preciso programa de reorganización del poblado, en el que participarán igualmente los campesinos. Hay que suponer su presencia como mano de obra en la realización de la muralla, y su intervención activa en la realiza- ción de las casas, que presentan una morfología idéntica en los cuatro casos excavados hasta el día de hoy (número total estimado unas I415). Destaca la ausencia de una casa morfológicamente diferenciada para el señor, o al menos de una torre o "cassero" (nombre que dan los documentos para designar las residencias señoriales en gran parte de la Toscana); tratándose de un área tan marginal, se puede pensar que el señor no tuviese residencia permanente ni ocasional allí. De hecho, los Maona, señores del valle, establecen su residencia en el castillo de Montecatini y más tarde en la ciudad de Lucca ${ }^{8}$.

En este caso pues, el elemento simbólico del poder señorial no viene ejercido por la torre, sino a través de las sólidas murallas que encierran el poblado. En otros castillos, como el ya señalado de Montecatini, son torres y murallas las que configuran el nuevo espacio medieval después del año 1000.

\section{El último cambio: la introducción de los ladrillos con módulos medievales}

El último aspecto que nos proponemos ilustrar, es la introducción de un nuevo material constructivo destinado a cambiar la imagen de la Toscana medieval: el ladrillo. Un elemento tan banal y aparentemente insignificante resulta de especial interés para comprender muchos problemas relativos a la arquitectura medieval toscana.

El siglo que va desde mediados del XII a mediados del XIII ve la reaparición de este material constructivo, que había sido muy empleado en el mundo clásico y había desaparecido en el altomedievo. Si bien se conservan evidencias de la continuidad productiva de las tégulas y otros ladrillos empleados para cubrir los edificios, los únicos ladrillos de construcción utilizados en el altomedievo son todos reutilizados y provenientes de estructuras clásicas (PARENTI, QUIRÓS CASTILLO 1996). A partir del siglo XII se observa la aparición de una serie de iglesias construidas exclusivamente en ladrillo en dos áreas toscanas:

8 Sobre la familia "de los Maona" SPICCIANI 1992. 
I. en Pisa a partir del XII comienzan a construirse las torres campanarias de los edificios religiosos en ladrillos (S. Sisto, S. Andrea, S. Frediano, S. Michele degli Scalzi) (QUIRÓS CASTILLO 1996b),

2. el área central de la Toscana siguiendo el trayecto de la Via Francesca o Via Francigena, verdadera autopista medieval que comunicaba Roma con Francia y que había sustituido las vías Aurelia y la Cassia a partir del período lombardo (MENUCCI 1995).

Nuestros estudios, tanto en la ciudad de Pisa como en el caso del área meridional de la Valdinievole han mostrado como las características dimensionales y técnicas de estos ladrillos son completamente nuevas y rompen con la tradición técnica presente hasta esta fecha. De otra parte, la heterogeneidad de estas producciones y su distribución territorial nos permite confirmar que se tratan de producciones autónomas, con centros productores construidos a pie de obra, sin posibilidad de que, por ahora, se produzca la transmisión y la extensión de la técnica, hecho que se consolidará solamente en el siglo siguiente.

S. Salvador de Fucecchio (I 190) y el Duomo de S. Miniato (I I80 ca.), situadas en el borde meridional de la Valdinievole, y la pieve de S. Juan de Monterapoli ( | 165), ligeramente más alejada, ilustran este proceso, evidenciando elementos arquitectónicos comunes que las relacionan, como son el módulo métrico del ladrillo $(27 / 30 \times 12 / 14 \times 5 / 6 \mathrm{~cm})$ la presencia de ladrillos esculpidos una vez ya cocidos con motivos a zigzag y el empleo de "bacini " 9 de producción "cobalto manganese" realizadas en Túnez durante toda la segunda mitad del siglo XII y los primerísimos decenios del XIII (BERTI, TONGIORGI 1972) ${ }^{10}$. Tiene especial interés la inscripción esculpida en el arquitrabe del pórtico de la pieve de Monterapoli, en la que se data la construcción, y se hace referencia a un "mais- ter Bonseri...vir probus ex gentes lombarda." (MORETTI, STOPANI 1982, p. 336)

Ya con el XIII se observa un cambio en la estructura de la producción, que empieza a hacerse muy intensa, con la creación de estructuras productivas estables, primero en ciudades como Pisa, Lucca y Siena, y ya en la segunda mitad del siglo en otras zonas (PARENTI, QUIRÓs CASTILLO 1996).

En la Valdinievole, el empleo del ladrillo es siempre marginal, pero resulta de especial interés como indicador del nivel de articulación de las estructuras productivas y del desarrollo urbanístico de los diversos centros. Cuando aparece en el siglo XII lo hace en un contexto eminentemente elitista, empleándose en construcciones eclesiásticas, y con estructuras productivas autónomas. Si bien en el siglo XIII su uso se hace extensivo en las áreas urbanas y comercialmente privilegiadas de la región, mantiene su carácter ciudadano y es un producto de lujo que raramente se encuentra en zonas rurales. En la Valdinievole aparece solamente en la segunda mitad del siglo XIV y exclusivamente en aquéllos castillos colinares ubicados en el área central del valle, situados al lado de la vía que unía las ciudades de Pistoia y Lucca (QUIRÓS CASTILLO 1995).

Uzzano, Pescia, Larciano, Montecatini o Buggiano, sólo por citar algunos de ellos, participan de este salto de calidad dentro de un proceso de crecimiento urbanístico relacionado con la presencia de mercados en estos centros, que se convierten en polos de atracción del poblamiento a partir de finales del siglo XIV y $X V$. Uno de ellos, Pescia, adquirirá el estatuto de ciudad.

En este sentido el ladrillo es fundamentalmente un indicador del desarrollo de una industria de materiales de construcción, coherente con el desarrollo de la industria local. Así pues,

\footnotetext{
9 El término "bacini” ha sido acuñado por G. B. Paseri en el año 1758 para referirse a una serie de formas cerámicas que habían sido colocadas en las fachadas y otras partes visibles de edificios, generalmente iglesias, con fines decorativos. Su presencia está testimoniada en todo el Mediterráneo, desde España hasta Grecia e Italia.

I0 Sobre los "bacini" de la iglesia de S. Miniato, BERTI, TONGIORGI I98Ia; como síntesis del problema de las cerámicas en arquitectura, ver el caso de la ciudad de Pisa, BERTI, TONGIORGI I98 Ib.
} 
el significado que puede darse a la producción y consumo de ladrillos en la Toscana medieval, salvo en el caso de los centros urbanos o de pequeñas ciudades (Volterra, San Gimignano, Certaldo,...) debe ser valorado con criterios cualitativos y no cuantitativos.

Por último, es necesario indicar como en la zona septentrional de la Valdinievole, solamente el crecimiento urbanístico de los siglos XVII y $X V I I I$ provocan la introducción de este material en la arquitectura local (QUIRÓS CASTILLO 1993).

\section{Conclusiones}

Actualmente existen dos tendencias paralelas y opuestas, que estudian las técnicas y los materiales constructivos y sus modificaciones. De un lado están los geógrafos, ciertos arqueólogos y naturalistas, que dan particular importancia al peso de los condicionamentos físicos a la hora de desarrollar un lenguaje constructivo y arquitectónico. Por otro lado, arquitectos tipologistas, etnógrafos e historiadores tienden a sobrevalorar el peso de los condicionantes culturales e históricos en estos procesos (DIANA 1989). Tal como muestra el caso de la Valdinievole, no es correcto usar determinismos geográficos o históricos para valorar aspectos aparentemente simples pero que adquieren un valor muy concreto si son situados en unas coordenadas históricas determinadas.

En la zona montañosa son muy escasos los recursos potencialmente útiles para la producción de ladrillos, pero es sobre todo la ausencia de un desarrollo de estos centros y de ciertas tipologías constructivas los que están a la base de que el ladrillo sea un producto tardío y marginal. El castillo de Montecarlo se construye en el año I333 completamente en ladrillo por parte de Lucca, quedando casi como un caso aislado en toda la Valdinievole durante este siglo (TORI 1984). Pero sucede que en el área donde se ubica Montecarlo no hay canteras cercanas de piedras para la construcción y sí buenas arcillas, a lo que hay que añadir la casi total ausencia en la primera mitad del XIV de movimiento de materiales constructivos.
En conclusión, como se ha intentado demostrar aquí, son muchos los criterios y las implicaciones que comporta el empleo de uno u otro material constructivo, incluso en un área rural como es la Valdinievole. A una arqueología histórica y metodológicamente abierta le corresponde el papel de superar los prejuicios actuales a la hora de elaborar el documento histórico.

\section{BIBLIOGRAFÍA}

ANDREOLLI B. (1983): Uomini nel medioevo. Studi sulla società lucchese dei secoli VIII-XI, Bolonia.

BERTI G., TONGIORGI L., (1972): "Ceramiche a cobalto e manganese su smalto bianco (Fine XII-inizio XIII)", en Atti del V Convegno Internazionale della Ceramica, Albisola, pp. |49-182.

BERTI G., TONGIORGI L., (198Ia): I bacini del Duomo di S. Miniato, Génova.

BERTI G., TONGIORGI L., ( 198 Ib): I bacini ceramici medievali delle chiese di Pisa, Roma.

BERTINI D., (|8|8): "Raccolta di documenti per servire alla storia ecclesiastica lucchese", en Memorie e documenti per servire all'istoria del Ducato di Lucca 4, Lucca.

BESSAC J. C., (1986): L'outillage traditionnel du tailleur de pierre, París.

BIANCHI G., (1993): "Segni lapidari nella Toscana centro-meridionale. Spunti per una ricerca", in Actes du Colloque International de Gliptographie de Hoepertingen (Belgique), 29 giugno-4 luglio 1992, Braine-le-Château, pp. 29-44.

BIANCHI G., ( 1995): "L'analisi dell'evoluzione di un sapere tecnico, per una rinnovata interpretazione dell'assetto abitativo e delle strutture edilizie del villaggio fortificato di Rocca S. Silvestro", en Acculturazione e mutamenti. Prospettive nell'archeologia medievale nel Mediterraneo, Florencia pp. 369-40।.

BIANCHI G., PARENTI R., (|99|): "Gli strumenti degli scalpellini toscani. Osservazioni preliminari", in "Le pietre nell'architettura: strutture e superfici." Atti del Convegno di Studi di Bressanone, 25-28 giugno 1991, Padua, pp. I39-149.

BOUGARD F., HUBERT E., NOYÉ G., (1987): "Les tecniches de construction en Sabine", Mélanges de l'École Française de Rome. Moyen Age, pp. 729-764.

BROGIOLO G. P. (ed.), (1994): "Edilizia residenziale tra $\checkmark$ e VIII secolo", 4 Seminario sul Tardoantico e l'Altomedioevo in Italia Settentrionale (Monte Barro-Galbiate, 2-4 settembre 1993), Padua.

CAMBI F., (1993): "Paesaggi d'Etruria e di Puglia", en Storia di Roma, vol. 3, L'età tardoantica, Roma, pp. 229-254. 
CIAMPOLTRINI G., ( 1995): "Ville, pievi, castelli: due schede archeologiche per l'organizzazione del territorio della Toscana nord-occidentale fra tarda antichità e alto medioevo", Archeologia Medievale XXII, pp. 557-568.

DIANA E., (1989): "Elementi del repertorio architettonico tardomedievale toscano. I casi di Montecarlo, Buggiano, Castelfranco di sopra, Figline e Vicchio", en D'una ville à l'autre. Structures matérielles et organisation de l'espace dans les villes européens (XIIle-XVle siècle), Collection de l'École Française de Rome 122, Roma, pp. 759-772.

FERRANDO I., GARDINI A., MANNONI T., (1978): "Zignago I: gli insediamenti e il territorio", Archeologia Medievale $\vee$, pp. 273-374

FRANCOVICH R., CUCINI C., PARENTI R., ( 1989): "Dalla "villa" al castello: dinamiche insediative e tecniche costruttive in Toscana fra tardoantico e bassomedioevo", Archeologia Medievale XVI, pp. 47-78.

MANCINI A.,(1986): Storia di Lucca, Lucca.

MANNONI T., CABONA D., FERRANDO CABONA I., (1988): "Archeologia globale del territorio. Metodi e risultati di una nuova strategia di ricerca in Liguria", en Castrum 2. Structures de l'habitat et occupation du sol dans les pays méditerranéens: les méthodes et l'apport de l'archéologie extensive, Roma, pp. 43-62.

MENUCCI A., (1995): Archeologia dell'architettura del Valdelsa, Università di Siena, tesis de licenciatura inédita.

MILANESE M. et alii (1996): Larciano. Museo e territorio, Roma, en prensa.

MILANESE M., QUIRÓS CASTILLO J. A. (1993): "Archeologia globale in Valdinievole", en Notiziario di archeologia medievale 6I, pp. 3-5.

MONTANARI M., (1979): L'alimentazione contadina nell'altomedioevo, Nápoles.

MORETTI I., STOPANI R., (1982): Italia Romanica. 5: La Toscana, Milán.

PARENTI R., QUIRÓS CASTILLO J. A., (1996): "La produzione dei mattoni della Toscana medievale (XII-XVI secolo). Un tentativo di sintesi", en La brique antique et médiévale: production et commercialisation d'un matériau (Paris, 27-29 octubre 1995), Roma, en prensa.

PESEZ J.-M., (1985): "Le renaissance de la construction en pierre après l'an Mil", en Pierre et métal dans le bâtiment au Moyen Âge. Études réunies par O. Chapelot et P. Benoit, Paris, pp. 197-217.

PUCCINELLI M. P., (1970): "La Valdinievole (Studio di geografia umana)" en Memorie della Società Geografica italiana XXIX, Roma.
QUIRÓS CASTILLO J. A., ( 1993): "Produzione di laterizi nell'alta Valdinievole: la Valleriana (XVII-XX sec.)" en Atti del Convegno su Pluriattività e mercati in Valdinievole (XVI-XIX secolo), Buggiano, pp. 139-I47.

QUIRÓS CASTILLO J. A., (1995): "Produzione di laterizi nella provincia di Pistoia e nella Toscana medievale e postmedievale", en Archeologia dell'architettura I, pp. 4I-5I.

QUIRÓS CASTILLO J. A., (1996a): Arqueología de un espacio de montaña: la Valleriana (Toscana, Italia), Departamento de Historia, Universidad de Oviedo,Tesina inédita.

QUIRÓS CASTILLO J. A., (1996b): "Le variazioni dimensionali dei mattoni da costruzione a Pisa nel medioevo e postmedioevo" en S. GELICHI, M. MILANESE (a cura di): Lo scavo dell'Arsenale Repubblicano di Pisa. La campagna 1993, en prensa.

QUIRÓS CASTILLO J. A., (1996c): "L'identità geografica-storica della Valdinievole alla luce dell'archeologia del territorio", en Atti del Convegno L'identità geografico-storica della Valdinievole, Buggiano, pp. 205-2I5.

QUIRÓS CASTILLO J. A., ZANCHETTA G., (1994): "Architettura e territorio nella Valleriana", en Atti del convegno Architettura in Valdinievole (s. $X-X X$ ), Buggiano pp. |57-174.

SETTIA A. A., (1984): Castelli e villaggi nell'Italia padana, Nápoles.

SPICCIANI A., (1992): "Una signoria rurale nel contado lucchese del secolo Xll: i da Buggiano e i da Maona", en Atti del convegno Signori e feudatari della Valdinievole dal $X$ al XII secolo, Buggiano pp. 43-76.

TORI G., ( 1984): "Maestro Lanfranco di Jacopo da Como ed i lavori alla rocca di Montecarlo del 1399-1400", Rivista di archeologia, storia, costume 3, pp. 27-48.

VANNI DESIDERI A., (1987): "La casa medievale del Poggio Salamartano", en Atti del Convegno L Abbazia di San Salvatore di Fucecchio e la Salamarza, Fucecchio, pp. 107-118.

VANNI DESIDERI A., ( 1989): "Bacini ceramici della chiesa abbaziale di S. Salvatore in Fucecchio (Firenze)", Notiziario di Archeologia Medievale 5I, p. 5.

VIOLANTE C., (1987): "I Traspadani in Tuscia nei secoli VIII e IX", en Studi di storia economica toscana nel Medioevo e nel Rinascimento in Memoria di Federigo Melis, Pisa. pp. $403-456$.

WICKHAM C., (|99|): "Aspetti socio-economici della Valdinievole nei secoli XI e XII" en Allucio da Pescia ( 1070 c.a-I I 34). Religione e società nei territori di Lucca e della Valdinievole, Roma, pp.279-296. 


\section{RESUMEN}

En el presente artículo se discute el significado histórico de las transformaciones de las técnicas y los materiales constructivos en la Toscana medieval. Para ello se estudia una subregión, la Valdinievole, situada en el centro de la Toscana septentrional, caracterizada por la ausencia de centros urbanos. La primera fase está representada por la desarticulación del sistema de poblamiento clásico, relacionado con la expansión de un modelo de hábitat basado en cabañas de madera. A partir del siglo XI se asiste a la reaparición del uso de la piedra en la arquitectura rural, en relación con los procesos de "incastellamento" y reorganización feudal del territorio. La última fase corresponde a la introducción, a partir de los siglos XII-XIII, del ladrillo medieval, primero en centros de consumo privilegiados, y posteriormente en otros ambitos rurales menores. 


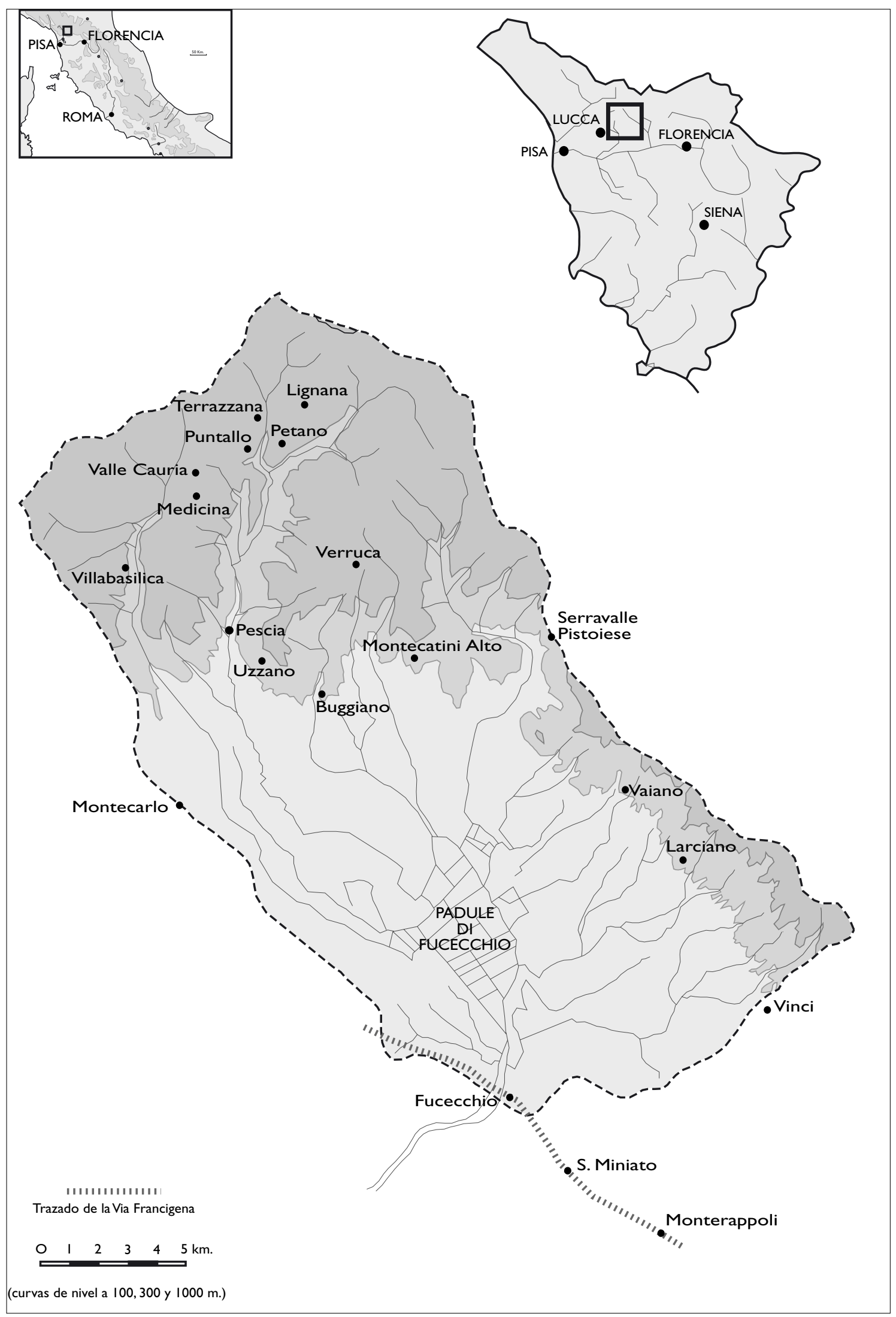

Fig. I. Ubicación de la Valdinievole, con indicaciones de las localidades citadas en el texto. 


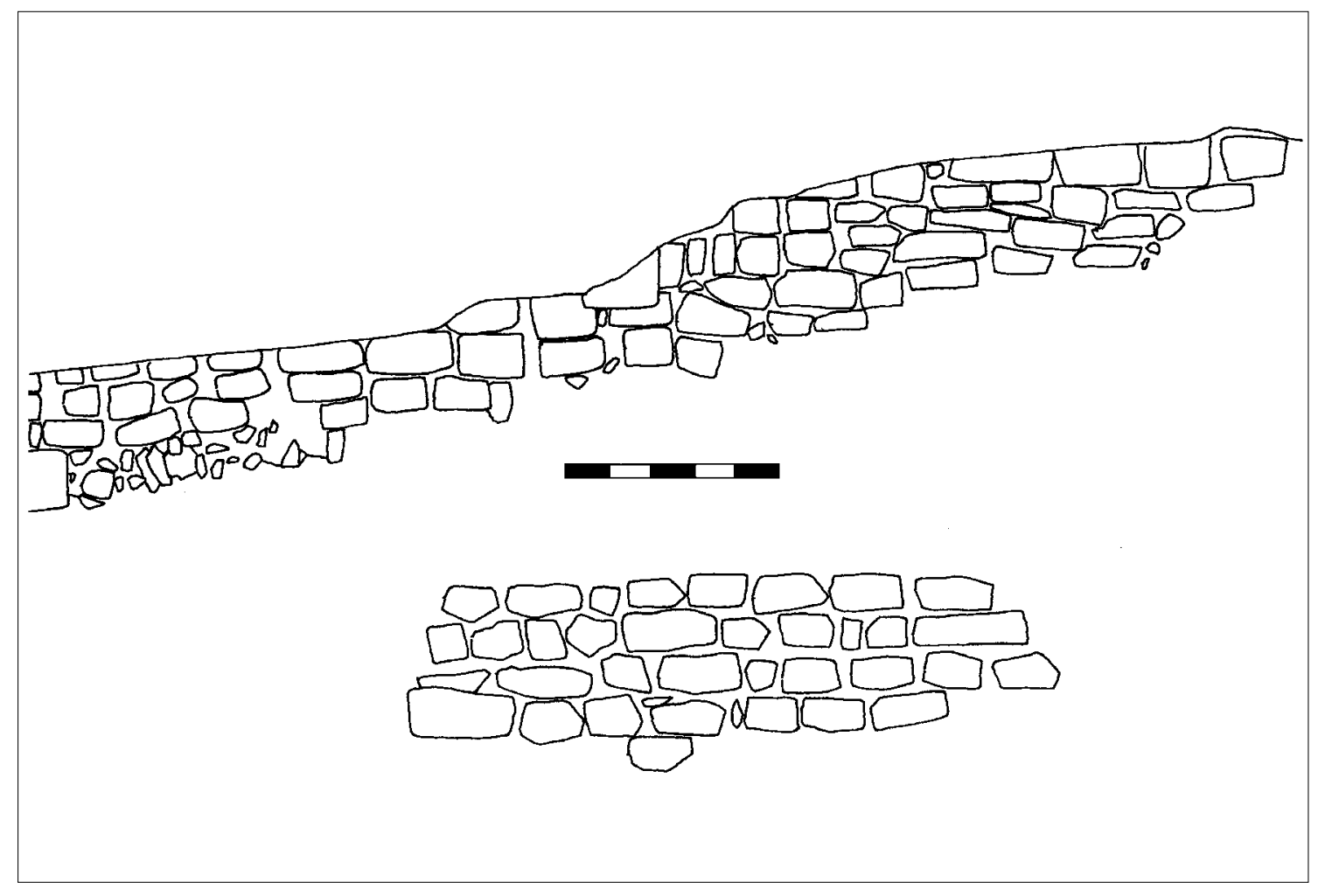

Fig. 2. Técnica constructiva de la muralla de Terrazzana.

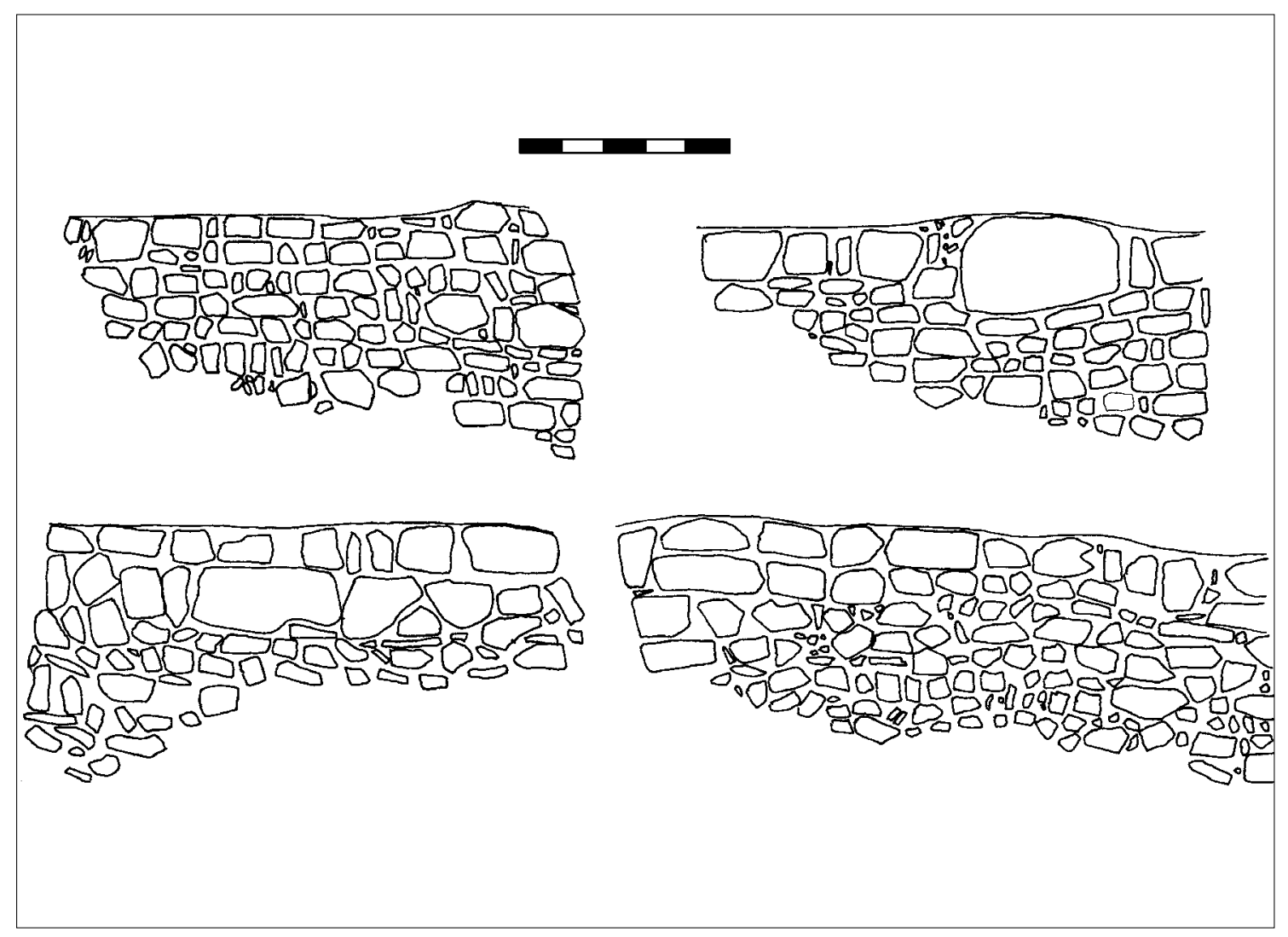

Fig. 3. Técnica constructiva de las casas de Terrazzana. 


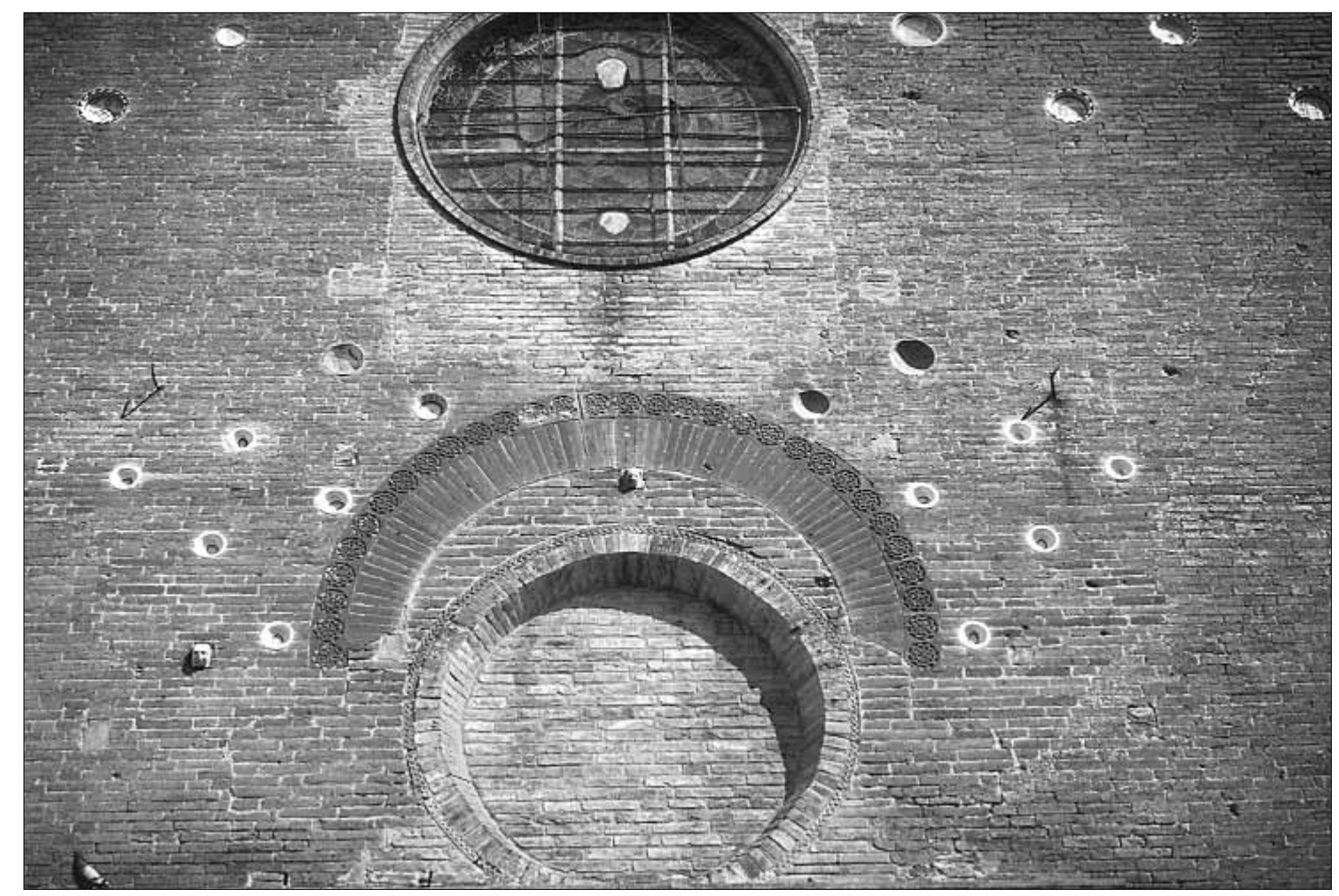

Fig. 4 Fachada del Duomo de S. Miniato (Pisa), con cerámicas producidas en Tunez.

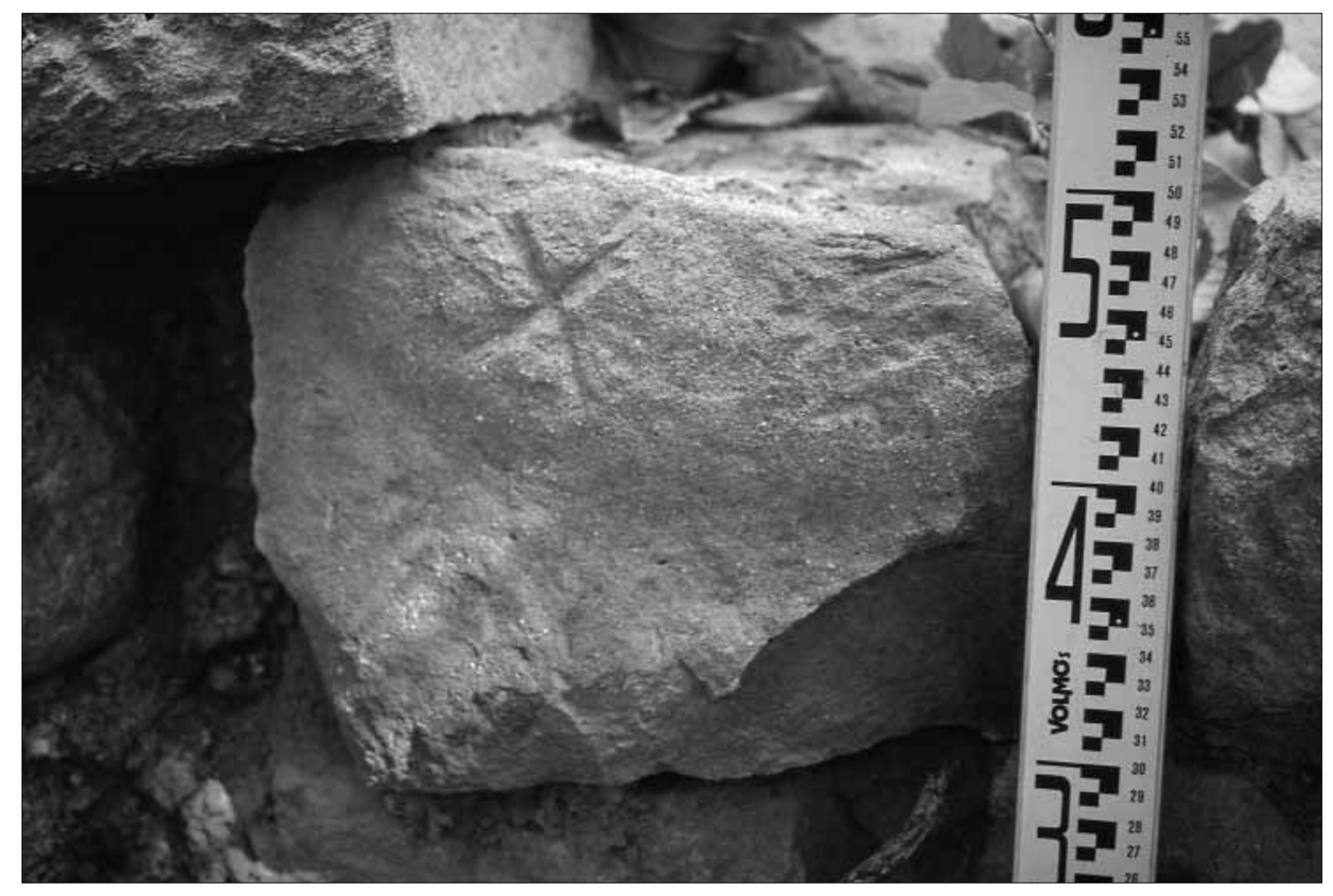

Fig. 5. Marca de cantero presente en la muralla de Terrazzana. 